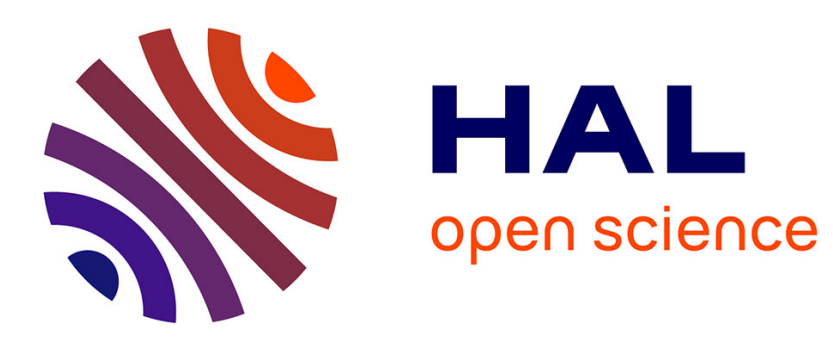

\title{
Forced Response Analysis of Integrally Bladed Disks With Friction Ring Dampers
}

Denis Laxalde, Fabrice Thouverez, Jean-Pierre Lombard

\section{To cite this version:}

Denis Laxalde, Fabrice Thouverez, Jean-Pierre Lombard. Forced Response Analysis of Integrally Bladed Disks With Friction Ring Dampers. Journal of Vibration and Acoustics, 2010, 132 (1), pp.011013. 10.1115/1.4000763 . hal-00453467

\section{HAL Id: hal-00453467 https://hal.science/hal-00453467}

Submitted on 4 Feb 2010

HAL is a multi-disciplinary open access archive for the deposit and dissemination of scientific research documents, whether they are published or not. The documents may come from teaching and research institutions in France or abroad, or from public or private research centers.
L'archive ouverte pluridisciplinaire HAL, est destinée au dépôt et à la diffusion de documents scientifiques de niveau recherche, publiés ou non, émanant des établissements d'enseignement et de recherche français ou étrangers, des laboratoires publics ou privés. 


\title{
Forced Response Analysis of Integrally Bladed Disks with Friction Ring Dampers
}

\author{
Denis Laxalde*, Fabrice Thouverez \\ École Centrale de Lyon \\ Laboratoire de Tribologie et Dynamique des Systèmes \\ 36 avenue Guy de Collongue, 69134 Ecully Cedex, France \\ Jean-Pierre Lombard \\ Snecma - Safran group \\ 77550 Moissy-Cramayel, France
}

\begin{abstract}
This paper investigates a damping strategy for integrally bladed disks (blisks) based on the use of friction rings. The steady-state forced response of the blisk with friction rings is derived using the so-called Dynamic Lagrangian Frequency-Time method adapted to cyclic structures with rotating excitations. In addition, an original approach for optimal determination of the number of Fourier harmonics is proposed. In numerical applications, a representative compressor blisk featuring several rings is considered. Each substructure is modeled using finite-elements and a reduced-order modeling technique is used for the blisk. The efficiency of this damping technology is investigated and friction dissipation phenomena are interpreted with respect to frequency responses. It is shown that friction damping effectiveness depends mainly on the level of dynamic coupling between blades and disk and on whether the dynamics features significant alternating stick / slip phases. Through parameter studies, design guidelines are also proposed.
\end{abstract}

\section{Introduction}

In turbomachineries, blisks (or integrally bladed disks) are single-piece rotating bladed disks either made as single components or assembled by welding blades on the disk. There are many advantages in using such components in place of traditional bladed disks assemblies, in particular in terms of aerodynamic performances or mass reduction. However, in contrast with assembled components, these single-piece-made structures no longer feature joints and friction interfaces (blade root, shrouds, under-platform dampers,...) and their internal damping is thus very low. Therefore, in severe operating conditions, blisks can experience high vibration levels and resonances leading to High Cycle Fatigue phenomena and in worst cases to failure. This, in particular, motivates the development of new vibration control devices.

The technology discussed in this paper provides an answer to this issue. It consists in introducing small circular rings underside the wheel of blisks. These are held in contact with the blisk due to centrifugal loads and friction dissipation can occur when some relative motions between rings and the blisk take place. Fig. 1 illustrates possible implantations of rings on a blisk cross section view. Similar friction damping devices are known to be efficient on other rotating structures such as labyrinth seals.

Friction damping devices in turbomachinery applications has been the subject of many research activities over the past decades and numerous methods of analysis can be found in the literature. The interested reader can find examples based on lumped parameter models or equivalent models in [1-3] and, more recently extended to more complex structures in [4-6]. The contact interface could be one dimensional or two-dimensional $[5,7,8]$. Besides, numerous experimental works were presented both in contact kinematic description $[9,10]$ and dynamic response prediction $[10-12]$.

As opposed to under-platform dampers in bladed disk assemblies, the friction ring dampers were seldom studied; Niemotka and Ziegert [13] have proposed a method of analysis and optimal design of split ring dampers

\footnotetext{
*Present affiliation: Department of Mechanical Engineering, McGill University, Montreal, QC, Canada. E-Mail: denis.laxalde@mcgill.ca
} 


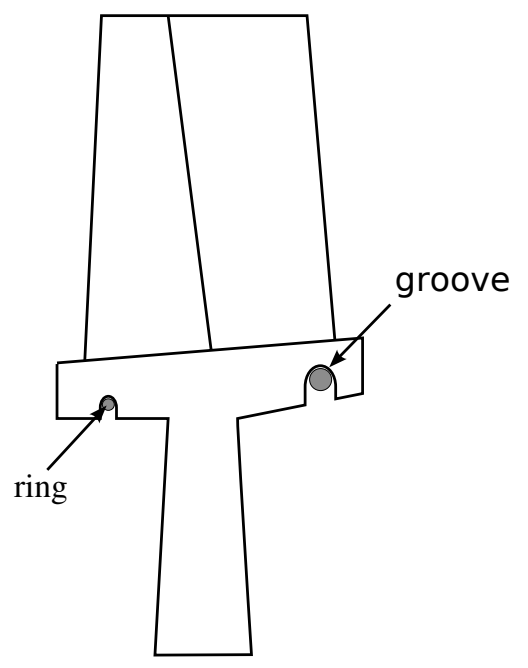

Figure 1: Cross-sectional view of a blisk with possible locations of friction rings

for seals based on quasi-static analysis and on one-dimensional models. More recently, studies in dynamics [14] where presented based on lumped parameters examples and lead mostly to qualitative results. The present paper proposes a numerical method adapted to $\mathrm{FE}$ models and presents some quantitative results. It is organized as follows. First, a numerical method for the computation of the steady-state forced response of a primary structure in frictional-contact with other substructures is proposed in a general framework. Emphasis is put on particular aspects such as cyclic symmetry and rotating excitation. In the framework of harmonic balance method with alternating frequency-time procedure, nonlinear forces forces are derived using a Dynamic Lagrangian Frequency-Time approach [5] which efficiently and accurately deals with non-smooth frictionalcontact laws. In a second part, results of numerical studies applied on a compressor blisk model are presented which demonstrates the efficiency of such damping devices.

\section{Nonlinear analysis}

The steady-state response of a system of cyclic structures coupled through frictional-contact forces and excited by a distribution of rotating forces is investigated. The solution strategy is based on a frequency-domain formulation of the equations of the motion (harmonic balance) which accounts for the specific aspects of rotating excitations and cyclic symmetry $[15,16]$ and takes advantages of the assumption that nonlinearities are cyclically symmetric. Reduction of the system equations on nonlinear interface degrees-of-freedom and relative displacements are also introduced. The contact formulation lies on the assumption of small deformations and small displacements of the structure and on matching meshes in the contact zones and thus enables the use of small sliding and node-to-node formalism. The computation of contact forces is performed using the Dynamic Lagrangian Frequency-Time method [5].

\subsection{Frequency-domain formulation}

Let us consider a system of $S$ linear substructures coupled through nonlinear forces. In a discretized formalism, the dynamical equation of motion of each substructure $s$ may be written as

$$
\boldsymbol{M}_{s} \ddot{\boldsymbol{u}}^{(s)}+\boldsymbol{C}_{s} \dot{\boldsymbol{u}}^{(s)}+\boldsymbol{K}_{s} \boldsymbol{u}^{(s)}=\boldsymbol{g}^{(s)}+\boldsymbol{f}^{(s)}
$$

In Eq. (1), $\boldsymbol{M}_{s}, \boldsymbol{C}_{s}$ and $\boldsymbol{K}_{s}$ refer to the mass, damping and stiffness structural matrices, $\boldsymbol{u}^{(s)}$ is the displacement degrees-of-freedom vector, $\boldsymbol{f}^{(s)}$ is an external forcing vector and $\boldsymbol{g}^{(s)}$ refers to nonlinear forces acting on each substructure. Note that as $\boldsymbol{g}^{(s)}$ may depend on displacements and velocities of several substructures.

Each substructure is rotationally periodic, that is, composed of $N_{s}$ identical sectors. The displacement degrees-of-freedom vector is written as:

$$
\boldsymbol{u}^{(s)}=\left[\boldsymbol{u}^{(s, 0)}, \boldsymbol{u}^{(s, 1)}, \ldots, \boldsymbol{u}^{\left(s, N_{s}-1\right)}\right]^{T}
$$


where $\boldsymbol{u}^{(s, j)}$ refers to the displacement vector of the $j$-th sector. In the following of this section, we focus on a single substructure and references to the substructure index $s$ are then omitted.

The displacement vector of a cyclic structure (as well as any other characteristic quantity) can be expressed in terms of so-called cyclic components $\left\{\boldsymbol{u}_{n}, n \in[0, \ldots, N-1]\right\}$ using discrete Fourier transforms:

$$
\boldsymbol{u}^{(j)}=\frac{1}{N} \sum_{n=0}^{N-1} \boldsymbol{u}_{n} e^{\mathrm{i} \frac{2 \pi j n}{N}} \quad \text { and } \quad \boldsymbol{u}_{n}=\sum_{j=0}^{N-1} \boldsymbol{u}^{(j)} e^{-\mathrm{i} \frac{2 \pi j n}{N}}
$$

or, introducing Fourier vectors: $\boldsymbol{e}_{n}=\left[1, e^{\mathrm{i} \frac{2 \pi n}{N}}, \ldots, e^{\mathrm{i} \frac{2 \pi n(N-1)}{N}}\right]^{T}$, in a more compact form:

$$
\boldsymbol{u}=\frac{1}{N} \sum_{n=0}^{N-1} \boldsymbol{e}_{n} \otimes \boldsymbol{u}_{n} \quad \text { and } \quad \boldsymbol{u}_{n}=\boldsymbol{e}_{n}^{*} \otimes \boldsymbol{u}
$$

A key feature of cyclic structure is that their structural matrices are block-circulant and can then be made block-diagonal by application of a discrete Fourier matrix composed of Fourier vectors $\left\{\boldsymbol{e}_{n}, n \in[0, N-1]\right\}$. Namely, if $\boldsymbol{A}$ denotes a structural matrix of the cyclic structure, we have for each $n \in[0, N-1]$

$$
\left(\boldsymbol{e}_{n}^{*} \otimes \boldsymbol{I}\right) \boldsymbol{A}\left(\boldsymbol{e}_{n} \otimes \boldsymbol{I}\right)=\boldsymbol{A}_{n}
$$

in which $\boldsymbol{I}$ is the identity matrix of the size of an elementary sector degrees-of-freedom vector and $\boldsymbol{A}_{n}$ is the $n$-th block (associated with cyclic harmonic $n$ ) of the so-called cyclic matrix.

Each cyclic structure is excited by a rotating harmonic force with order $p$ and angular frequency $\omega=p \Omega$ ( $\Omega$ denotes the angular velocity of the rotor). This force can be expressed [17] as a rotating component defined for each sector $j$ as

$$
\boldsymbol{f}^{(j)}(t)=\hat{\boldsymbol{f}}_{1} e^{\mathrm{i}\left(\omega t+\frac{2 \pi j p}{N}\right)}
$$

in which $\hat{\boldsymbol{f}}_{1}$ refers to the complex amplitude vector of the first (and only) harmonic of the excitation and is defined on the so-called reference sector of the cyclic structure.

We are interested in the derivation of synchronous periodic solutions of Eq. (1). Due to the rotating excitation and the nonlinearities, these solutions are assumed to be traveling waves with multiple harmonic components. Accordingly, the solution vector $\boldsymbol{u}(t)$ is written in the form of a truncated Fourier series:

$$
\boldsymbol{u}^{(j)}(t)=\sum_{k=-N_{h}}^{N_{h}} \hat{\boldsymbol{u}}_{k} e^{\mathrm{i} k\left(\omega t+\frac{2 \pi j p}{N}\right)}=\sum_{k=-N_{h}}^{N_{h}} \hat{\boldsymbol{u}}_{k} e^{\mathrm{i}\left(k \omega t+\frac{2 \pi j n}{N}\right)} \quad \text { with } n \equiv k p \quad(\bmod N)
$$

where $\hat{\boldsymbol{u}}_{k} \in \mathbb{C}$ is $k$-th harmonic component of the response. From Eq. (6), it appears that each harmonic $k$ corresponds to a cyclic harmonic $p k$ (with reference to Eqs. (3)) which, due to the aliasing of the discrete Fourier series, actually coincides with a fundamental cyclic harmonics $n \equiv p k(\bmod N)$.

In Eq. (1), the nonlinear forces vector $\boldsymbol{g}$ depends on the displacement and velocity vectors which, according to Eq. (6), are composed of cyclic super-harmonics of the fundamental harmonic of the excitation $n \equiv k p$ $(\bmod N)$. It is here assumed that these nonlinear forces are also symmetric and hence do not bring additional cyclic harmonics:

$$
\forall m \not \equiv p k \quad(\bmod N), \quad \sum_{j=0}^{N-1} \boldsymbol{g}^{(j)} e^{-\mathrm{i} \frac{2 \pi m j}{N}}=0
$$

With this assumption, nonlinear forces can be written similarly to Eq. (6):

$$
\boldsymbol{g}^{(j)}=\sum_{k=-N_{h}}^{N_{h}} \hat{\boldsymbol{g}}_{k} e^{\mathrm{i}\left(\omega t+\frac{2 \pi j n}{N}\right)} \quad \text { with } \quad \hat{\boldsymbol{g}}_{k}=\frac{1}{T} \int_{T} \sum_{j=0}^{N-1} \boldsymbol{g}^{(j)} e^{-\mathrm{i}\left(k \omega t+\frac{2 \pi j n}{N}\right)} \mathrm{d} t
$$

The frequency-domain form of Eq. (1) can then be derived by introducing Eqs. (6) and (8) in the latter and performing a Fourier-Galerkin projection with respect to time

$$
\left(-(k \omega)^{2} \boldsymbol{M}+\mathrm{i} k \omega \boldsymbol{C}+\boldsymbol{K}\right) \boldsymbol{e}_{n} \otimes \hat{\boldsymbol{u}}_{k}=\boldsymbol{e}_{n} \otimes\left(\hat{\boldsymbol{f}}_{k}+\hat{\boldsymbol{g}}_{k}\right) \quad \forall k \in\left[-N_{h}, N_{h}\right]
$$


and a discrete Fourier transform with respect to cyclic harmonics (pre-multiplication by $\boldsymbol{e}_{n}^{*} \otimes \boldsymbol{I}$ )

$$
\left(-(k \omega)^{2} \boldsymbol{M}_{n}+\mathrm{i} k \omega \boldsymbol{C}_{n}+\boldsymbol{K}_{n}\right) \hat{\boldsymbol{u}}_{k}=\hat{\boldsymbol{f}}_{k}+\hat{\boldsymbol{g}}_{k} \quad \forall k \in\left[-N_{h}, N_{h}\right]
$$

Each harmonic $k$ of the response is associated with a cyclic harmonic $n \equiv p k(\bmod N)$, which is involved in the definition of the dynamic stiffness $\boldsymbol{Z}_{n}(k \omega)=-(k \omega)^{2} \boldsymbol{M}_{n}+\mathrm{i} k \omega \boldsymbol{C}_{n}+\boldsymbol{K}_{n}$. All harmonics are a priori coupled due to the nonlinear terms $\hat{\boldsymbol{g}}_{k}(\hat{\boldsymbol{u}})$.

Gathering all equations in (10), the frequency-domain nonlinear system can be written in a compact form:

$$
\boldsymbol{Z}(\omega) \hat{\boldsymbol{u}}=\hat{\boldsymbol{f}}+\hat{\boldsymbol{g}}(\hat{\boldsymbol{u}})
$$

in which

$$
\begin{gathered}
\boldsymbol{Z}=\operatorname{diag}\left(\boldsymbol{Z}_{n}(k \omega), k \in\left[-N_{h}, N_{h}\right]\right) \\
\hat{\boldsymbol{u}}=\left[\hat{\boldsymbol{u}}_{-N_{h}}, \ldots, \hat{\boldsymbol{u}}_{0}, \ldots, \hat{\boldsymbol{u}}_{N_{h}}\right], \quad \hat{\boldsymbol{g}}=\left[\hat{\boldsymbol{g}}_{-N_{h}}, \ldots, \hat{\boldsymbol{g}}_{0}, \ldots, \hat{\boldsymbol{g}}_{N_{h}}\right] \quad \text { and } \hat{\boldsymbol{f}}=\left[\hat{\boldsymbol{f}}_{-N_{h}}, \ldots, \hat{\boldsymbol{f}}_{0}, \ldots, \hat{\boldsymbol{f}}_{N_{h}}\right]
\end{gathered}
$$

In the following, distinctions between substructures are further introduced so that Eq. (11) is rewritten for each substructure $s \in[1, \ldots, S]$ :

$$
\boldsymbol{Z}_{s} \hat{\boldsymbol{u}}^{(s)}=\hat{\boldsymbol{f}}^{(s)}+\hat{\boldsymbol{g}}^{(s)}
$$

Recalling that $\hat{\boldsymbol{g}}^{(s)}, s \in[1, \ldots, S]$ are nonlinear coupling forces between substructures, it is then of interest to reduce the dimension of the nonlinear systems (14) taking into account that: (a) nonlinear forces only act on boundary degrees-of-freedom and (b) nonlinear forces involve relative displacements and velocities.

\subsection{Reduction on nonlinear degrees-of-freedom and relative displacements}

Nonlinear forces are located at interfaces between substructures so that they actually act on a small number of degrees-of-freedom (called nonlinear degrees-of-freedom). Let us consider substructure $s$ which displacement and forces vectors are partitioned according to nonlinear degrees-of-freedom $\cdot(s, n)$ and linear degrees-of-freedom ${ }^{(s, l)}$. Namely, for the displacement vectors, we have:

$$
\hat{\boldsymbol{u}}^{(s)}=\boldsymbol{B}_{s, n} \hat{\boldsymbol{u}}^{(s, n)}+\boldsymbol{B}_{s, l} \hat{\boldsymbol{u}}^{(s, l)}
$$

in which $\boldsymbol{B}_{s, n}$ and $\boldsymbol{B}_{s, l}$ are Boolean matrices localizing nonlinear and linear degrees-of-freedom respectively. Nonlinear forces are zero on internal degrees-of-freedom:

$$
\hat{\boldsymbol{g}}^{(s, l)}=\boldsymbol{B}_{s, l}^{T} \hat{\boldsymbol{g}}^{(s)}=0
$$

Accordingly, Eq. (14) can be split into two equations

$$
\begin{aligned}
\boldsymbol{B}_{s, n}^{T} \boldsymbol{Z}_{s} \boldsymbol{B}_{s, n} \hat{\boldsymbol{u}}^{(s, n)}+\boldsymbol{B}_{s, n}^{T} \boldsymbol{Z}_{s} \boldsymbol{B}_{s, l} \hat{\boldsymbol{u}}^{(s, l)}=\boldsymbol{B}_{s, n}^{T} \hat{\boldsymbol{f}}^{(s)}+\boldsymbol{B}_{s, n}^{T} \hat{\boldsymbol{g}}^{(s)} \\
\boldsymbol{B}_{s, l}^{T} \boldsymbol{Z}_{s} \boldsymbol{B}_{s, n} \hat{\boldsymbol{u}}^{(s, n)}+\boldsymbol{B}_{s, l}^{T} \boldsymbol{Z}_{s} \boldsymbol{B}_{s, l} \hat{\boldsymbol{u}}^{(s, l)}=\boldsymbol{B}_{s, l}^{T} \hat{\boldsymbol{f}}^{(s)}
\end{aligned}
$$

Using Eq. (17b), $\hat{\boldsymbol{u}}^{(s, l)}$ can then be eliminated from Eq. (17a) leading to reduced equations

$$
\boldsymbol{Z}_{s, r} \hat{\boldsymbol{u}}^{(s, r)}=\hat{\boldsymbol{f}}^{(s, r)}+\hat{\boldsymbol{g}}^{(s, r)}
$$

in which

$$
\begin{gathered}
\boldsymbol{Z}_{s, r}=\boldsymbol{B}_{s, n}^{T} \boldsymbol{Z}_{s} \boldsymbol{B}_{s, n}-\left(\boldsymbol{B}_{s, l}^{T} \boldsymbol{Z}_{s} \boldsymbol{B}_{s, l}\right)^{-1} \boldsymbol{B}_{s, n}^{T} \boldsymbol{Z}_{s} \boldsymbol{B}_{s, l} \\
\hat{\boldsymbol{f}}^{(s, r)}=\left(\boldsymbol{B}_{s, n}^{T}-\left(\boldsymbol{B}_{s, l}^{T} \boldsymbol{Z}_{s} \boldsymbol{B}_{s, l}\right)^{-1} \boldsymbol{B}_{s, n}^{T} \boldsymbol{Z}_{s} \boldsymbol{B}_{s, l} \boldsymbol{B}_{s, l}^{T}\right) \hat{\boldsymbol{f}}^{(s)} \\
\hat{\boldsymbol{g}}^{(s, r)}=\boldsymbol{B}_{s, n}^{T} \hat{\boldsymbol{g}}^{(s)} \text { and } \hat{\boldsymbol{u}}^{(s, r)}=\boldsymbol{B}_{s, n}^{T} \hat{\boldsymbol{u}}^{(s)}
\end{gathered}
$$

Finally, gathering all reduced systems (18) a global reduced system can be written as

$$
\boldsymbol{Z}_{r} \hat{\boldsymbol{u}}^{(r)}=\hat{\boldsymbol{f}}^{(r)}+\hat{\boldsymbol{g}}^{(r)}
$$


in which $\boldsymbol{Z}_{r}$ is a block-diagonal matrix made of each block $\boldsymbol{Z}_{s, r}$ and vectors $\hat{\boldsymbol{u}}^{(r)}, \hat{\boldsymbol{f}}^{(r)}$ and $\hat{\boldsymbol{g}}^{(r)}$ are concatenations of each substructure reduced vectors $\hat{\boldsymbol{u}}^{(s, r)}, \hat{\boldsymbol{f}}^{(s, r)}$ and $\hat{\boldsymbol{g}}^{(s, r)}$ respectively.

The coupling between substructures is typically defined using an equilibrium equation involving interaction forces $\hat{\boldsymbol{g}}^{s}, s \in[1, \ldots, S]$ and which can be formally written as:

$$
\boldsymbol{L}^{T} \hat{\boldsymbol{g}}^{(r)}=0
$$

The matrix $\boldsymbol{L}$ is Boolean (in case of conformable interface meshes) and defined such that Eq. (21) gathers each interface equilibrium equation. For example, considering two given substructures $\alpha$ and $\beta$, Eq. (21) states that the resultant of interfaces forces is zero: $\hat{\boldsymbol{g}}^{\alpha, r}+\hat{\boldsymbol{g}}^{\beta, r}=0$. A unique set of interface forces $\hat{\boldsymbol{\lambda}}$ can then be defined by the following relation

$$
\hat{\boldsymbol{g}}^{(r)}=\boldsymbol{P} \hat{\boldsymbol{\lambda}}
$$

in which matrix $\boldsymbol{P}$ is defined such as $\boldsymbol{P}^{T}=$ null $\left(\boldsymbol{L}^{T}\right)$. Accordingly, the vector of interface relative displacements $\hat{\boldsymbol{q}}$ can be defined as

$$
\hat{\boldsymbol{q}}=\boldsymbol{P}^{T} \hat{\boldsymbol{u}}^{(r)}
$$

Following the previous example, restrictions of vectors $\hat{\boldsymbol{\lambda}}$ and $\hat{\boldsymbol{q}}$ to substructures $\alpha$ and $\beta$ are respectively: $\hat{\boldsymbol{g}}^{\beta, r}\left(=-\hat{\boldsymbol{g}}^{\alpha, r}\right)$ and $\hat{\boldsymbol{u}}^{(\beta, r)}-\hat{\boldsymbol{u}}^{(\alpha, r)}$. Finally, introducing Eqs. (23) and (22) in Eq. (20) and performing some algebraic calculations leads to the frequency-domain dynamical equation reduced in nonlinear relative coordinates:

$$
\boldsymbol{\Lambda}(\omega) \hat{\boldsymbol{q}}=\hat{\boldsymbol{\eta}}+\hat{\boldsymbol{\lambda}}(\hat{\boldsymbol{q}})
$$

in which the reduced dynamical stiffness and forces are

$$
\boldsymbol{\Lambda}=\left(\boldsymbol{P}^{T} \boldsymbol{Z}_{r}^{-1} \boldsymbol{P}\right)^{-1} \quad \text { and } \quad \hat{\boldsymbol{\eta}}=\boldsymbol{\Lambda} \boldsymbol{P}^{T} \boldsymbol{Z}_{r}^{-1} \hat{\boldsymbol{f}}^{(r)}
$$

Eq. (24) defines a nonlinear algebraic system which can be solved using a Newton-like algorithm. The derivation of nonlinear forces $\hat{\boldsymbol{\lambda}}$ in the frequency-domain as functions of the frequency-domain displacements $\hat{\boldsymbol{q}}$ will be explained in the following section.

\subsection{Contact forces calculation}

Solving Eq. (24) requires prior computations of the nonlinear forces vector $\hat{\boldsymbol{\lambda}}$ as a function of the multi-harmonic displacement vector $\hat{\boldsymbol{q}}$. To undertake this issue, the use of Alternating Frequency-Time (AFT) method [18] is common and good performances are obtained if combined with discrete Fourier transforms (DFT). This procedure typically consists in (1) expressing displacements and velocities in the time-domain from the multiharmonic displacement vector using an inverse DFT, (2) using a nonlinear (time-domain) operator to derive nonlinear forces accordingly, (3) transforming the latter in the frequency domain using a forward DFT.

Time-domain computations of the contact and friction forces are also challenging since these are defined by non-smooth functions of displacements and velocities. Concerning Coulomb friction in particular, several approaches have been proposed. Regularized expressions of the sign function have been proposed in [11, 19]; this enables direct (non-iterative) computations of friction forces in the time domain. Penalty stiffnesses are widely used to soften the contact interfaces $[4,6,15]$. These strategies often require an iterative computation of the friction forces in the time domain where the separation/stick/slip transitions have to be determined at each step. Nacivet et al. [5] have proposed an original strategy - named the Dynamic Lagrangian FrequencyTime method (DLFT) - based on augmented Lagrangian to deal with non-smooth contact-friction laws. This method was later used for the study of friction mechanisms in blade attachments [20]. In the present study, a similar approach is used but the formulation is presented using a mixture of relative displacement in the normal direction and relative velocity for the tangential directions of the contact interface. This makes it possible to derive the time-domain nonlinear forces directly (i.e. in a non-iterative manner).

\section{Penalty function in the frequency-domain}

Inspired by augmented Lagrangian formulations, the dynamic Lagrangian method introduces a penalty functional derived from the frequency-domain equation (24). The following function defines the Lagrange multiplier (i.e. nonlinear forces) as:

$$
\hat{\boldsymbol{\lambda}}=\boldsymbol{\Lambda} \hat{\boldsymbol{q}}-\hat{\boldsymbol{\eta}}+\varepsilon_{t} \boldsymbol{L}_{t}\left(\nabla \boldsymbol{L}_{t}^{T} \hat{\boldsymbol{q}}-\hat{\boldsymbol{V}}_{t}\right)+\varepsilon_{n} \boldsymbol{L}_{n}\left(\boldsymbol{L}_{n}^{T} \hat{\boldsymbol{q}}-\hat{\boldsymbol{X}}_{n}\right)
$$


In the latter equation, apart from quantities already in Eq. (24), appear two penalty terms which respectively refer to tangential and normal directions at the contact interfaces and are identified by subscripts $t$ and $n$. Matrices $\boldsymbol{L}_{t}$ and $\boldsymbol{L}_{n}$ are Boolean matrices which restrict the degrees-of-freedom vector $\hat{\boldsymbol{q}}$ to tangential (2 components) and normal (1 component) directions. These penalty terms feature two new vectors $\hat{\boldsymbol{V}}_{t}$ and $\hat{\boldsymbol{X}}_{n}$, respectively representing relative tangential velocities and relative normal displacements. These vectors are determined through an Alternating Frequency-Time procedure in order to fulfill the frictional contact constraints and their time-domain counterparts aim at being identical to actual relative velocities and displacements (derived from $\hat{\boldsymbol{q}}$ ). Matrix $\nabla$ is a time-differential operator in the frequency domain. To each penalty term, is associated a penalty coefficient, $\varepsilon_{t}$ and $\varepsilon_{n}$ respectively. A good choice for these parameters consists in ensuring that penalty terms balance with stiffness terms (Charleux et al. [20] suggested to use a value close to the spectral radius of $\boldsymbol{\Lambda}(\omega)$ ).

The key point of the dynamic Lagrangian approach is that combining Eqs. (24) and (26) results in

$$
\begin{aligned}
& \varepsilon_{t}\left(\nabla \boldsymbol{L}_{t}^{T} \hat{\boldsymbol{q}}-\hat{\boldsymbol{V}}_{t}\right)=0 \\
& \varepsilon_{n}\left(\boldsymbol{L}_{n}^{T} \hat{\boldsymbol{q}}-\hat{\boldsymbol{X}}_{n}\right)=0
\end{aligned}
$$

Thus proper derivations of vectors $\hat{\boldsymbol{V}}_{t}$ and $\hat{\boldsymbol{X}}_{n}$ will ensure that both the equation of motion and the contact constraints are verified at convergence.

\section{Prediction / correction in the time-domain}

The AFT procedure aims at determining Lagrange multipliers $\hat{\boldsymbol{\lambda}}$ along with vectors $\hat{\boldsymbol{V}}_{t}$ and $\hat{\boldsymbol{X}}_{n}$. A global summary of the procedure is given in Fig. 2. First, Eq. (26) is reformulated in order to separate terms which depends on the "known" (within prediction from Newton iterations) Fourier components $\hat{\boldsymbol{q}}$ from other quantities to be determined:

$$
\hat{\boldsymbol{\lambda}}=\hat{\boldsymbol{\lambda}}_{q}(\hat{\boldsymbol{q}})-\hat{\boldsymbol{\lambda}}_{X}\left(\hat{\boldsymbol{V}}_{t}, \hat{\boldsymbol{X}}_{n}\right)
$$

Terms dependent of $\hat{\boldsymbol{q}}$ are in $\hat{\boldsymbol{\lambda}}_{q}$ and those dependent of $\left(\hat{\boldsymbol{V}}_{t}, \hat{\boldsymbol{X}}_{n}\right)$ are in $\hat{\boldsymbol{\lambda}}_{X}$, which has to be calculated, in the time domain, using contact and friction laws namely, contact or separation in the normal direction and stick / slip in the tangent direction.

Second, Eq. (28) is transformed in the time-domain using an inverse discrete Fourier transform. Focusing on one contact element (a pair of nodes, in the node-to-node formulation), we have the time-domain Lagrange multiplier, for the $k$-th time-step,

$$
{ }^{k} \overline{\boldsymbol{\lambda}}={ }^{k} \overline{\boldsymbol{\lambda}}_{q}-{ }^{k} \overline{\boldsymbol{\lambda}}_{X}, \quad k \in[1, \ldots, m]
$$

A prediction / correction procedure is then used to compute the contact forces ${ }^{k} \bar{\lambda}$ in accordance with the contact and friction laws. For each time step, the prediction is done assuming that the contact element is stuck, which means that ${ }^{k} \overline{\boldsymbol{V}}_{t}=0$ and ${ }^{k} \bar{X}_{n}=0$ and thus according Eq. (29),

$$
{ }^{k} \overline{\boldsymbol{\lambda}}_{\text {pre }}={ }^{k} \overline{\boldsymbol{\lambda}}_{q}
$$

Next, the value of ${ }^{k} \overline{\boldsymbol{\lambda}}_{X}$ is derived to correct ${ }^{k} \overline{\boldsymbol{\lambda}}$ depending on the contact state:

1. Separation: ${ }^{k} \bar{\lambda}_{\text {pre,n } n}^{N} \geq N_{0}$ (where $N_{0}$ is the normal preload due to the engine rotation). The contact is lost, then the normal contact force should be zero and consequently:

$$
{ }^{k} \overline{\boldsymbol{\lambda}}_{X}={ }^{k} \overline{\boldsymbol{\lambda}}_{q}
$$

2. Stick: ${ }^{k} \bar{\lambda}_{p r e, n}<N_{0}$ and $\left\|{ }^{k} \overline{\boldsymbol{\lambda}}_{\text {pre }, t}\right\|<\left.\mu\right|^{k} \bar{\lambda}_{\text {pre }, n} \mid$.

In this case, the relative normal displacement and tangential velocity are zero and the prediction is correct, hence,

$$
{ }^{k} \overline{\boldsymbol{\lambda}}_{X}=0
$$

3. Slip: ${ }^{k} \bar{\lambda}_{\text {pre }, n}<N_{0}$ and $\left\|^{k} \overline{\boldsymbol{\lambda}}_{\text {pre }, t}\right\|>\left.\mu\right|^{k} \bar{\lambda}_{\text {pre }, n} \mid$.

There, the relative normal displacement is still zero, leading to

$$
{ }^{k} \bar{\lambda}_{X, n}=0
$$


whereas, in the tangential direction, the Coulomb law can be written as

$$
{ }^{k} \overline{\boldsymbol{\lambda}}_{t}=\left.\mu\right|^{k} \bar{\lambda}_{p r e, n} \mid \frac{{ }^{k} \overline{\boldsymbol{V}}_{t}}{\left\|{ }^{k} \overline{\boldsymbol{V}}_{t}\right\|}
$$

which, recalling that $\overline{\boldsymbol{\lambda}}_{X, t}=\epsilon_{t} \overline{\boldsymbol{V}}_{t}$, can be rewritten as,

$$
{ }^{k} \overline{\boldsymbol{\lambda}}_{t}=\left.\mu\right|^{k} \bar{\lambda}_{p r e, n} \mid \frac{{ }^{k} \overline{\boldsymbol{\lambda}}_{X, t}}{\left\|{ }^{k} \overline{\boldsymbol{\lambda}}_{X, t}\right\|}
$$

and combining this last equation with Eqs. (30) and (29), we obtain,

$$
{ }^{k} \overline{\boldsymbol{\lambda}}_{p r e, t}=\left(\mu \frac{{ }^{k} \bar{\lambda}_{p r e, n} \mid}{\left\|{ }^{k} \overline{\boldsymbol{\lambda}}_{X, t}\right\|}+1\right){ }^{k} \overline{\boldsymbol{\lambda}}_{X, t}
$$

which proves that ${ }^{k} \overline{\boldsymbol{\lambda}}_{X, t}$ and ${ }^{k} \overline{\boldsymbol{\lambda}}_{\text {pre,t }}$ are colinear. We finally obtain,

$$
{ }^{k} \overline{\boldsymbol{\lambda}}_{t}=\left.\mu\right|^{k} \bar{\lambda}_{p r e, n} \mid \frac{{ }^{k} \overline{\boldsymbol{\lambda}}_{\text {pre }, t}}{\left\|{ }^{k} \overline{\boldsymbol{\lambda}}_{p r e, t}\right\|}
$$

and consequently,

$$
{ }^{k} \overline{\boldsymbol{\lambda}}_{X, t}={ }^{k} \overline{\boldsymbol{\lambda}}_{p r e, t}\left(1-\mu \frac{\left|{ }^{k} \bar{\lambda}_{p r e, n}\right|}{\left\|{ }^{k} \overline{\boldsymbol{\lambda}}_{p r e, t}\right\|}\right)
$$

The final step simply consists in transforming the updated Lagrange multipliers in the frequency domain using a forward DFT.

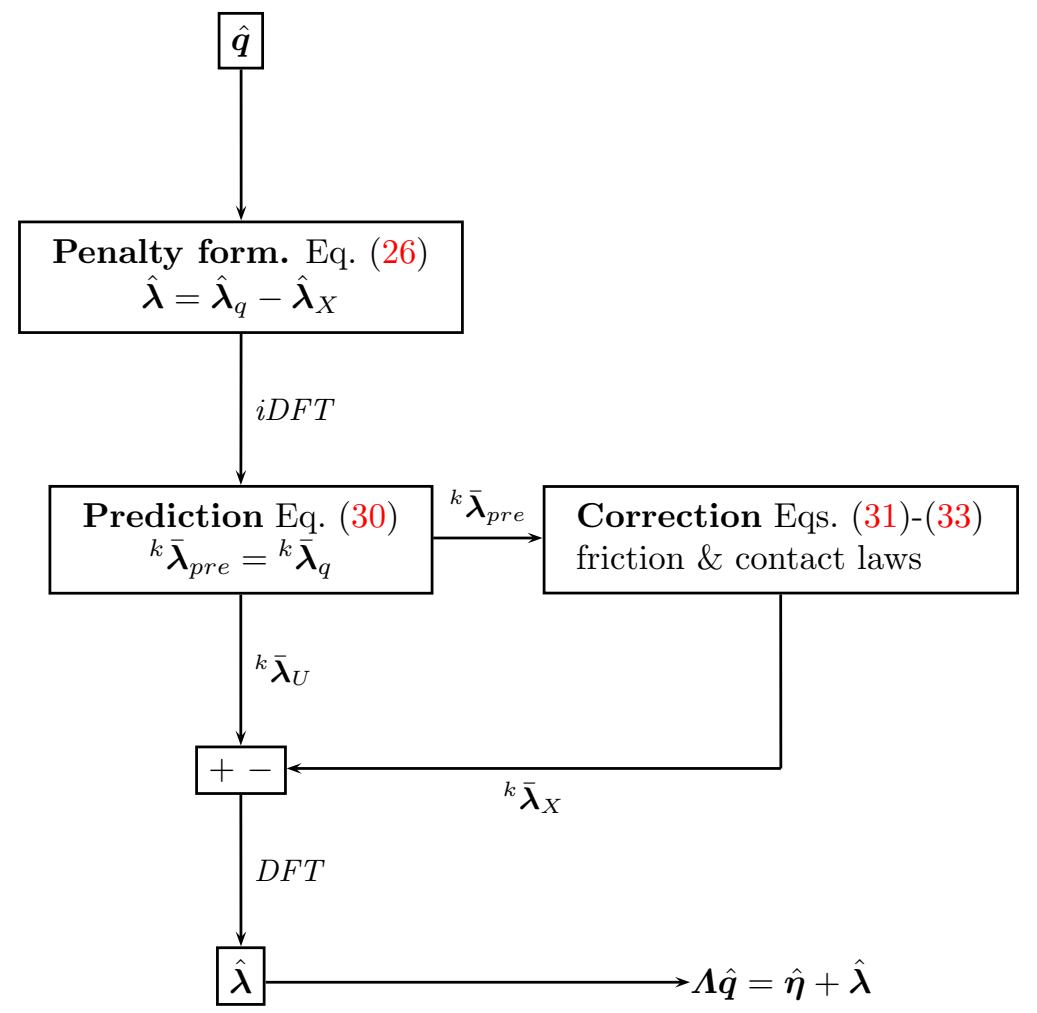

Figure 2: Computation of the Lagrange multiplier vector 
Remark on the number of harmonics: As Fourier methods are used, the proper choice of the number of harmonics to be retained to correctly approximate the response is a recurrent issue. In any approach that uses alternating frequency-time procedure, it is possible to dynamically update the number of harmonics by monitoring the error between the "exact" time-domain nonlinear forces and the approximated multi-harmonics Fourier series:

$$
\varepsilon\left(N_{h}\right)=\sum_{k=1}^{m}\left({ }^{k} \overline{\boldsymbol{\lambda}}-\frac{1}{N_{h}} \sum_{n=0}^{N_{h}-1} \hat{\boldsymbol{\lambda}}_{n} e^{\mathrm{i} \frac{2 \pi k n}{N_{h}}}\right)
$$

In a frequency-response analysis, this error criterion will naturally increasing around resonances if the number of harmonics is kept constant due to the growth of nonlinear effects with the vibratory amplitude. Thus during frequency sweepings, one can simply increase or decrease the number of harmonics $N_{h}$ from one frequency step to an other in order to keep the criterion of Eq. (34) constant.

\section{$3 \quad$ Numerical applications}

In this section, the computational method previously presented is applied to forced response analysis of a blisk with one or several friction ring dampers. Several parameter studies are presented to illustrate the efficiency of this damping technology depending on the excitation configuration and on the damper characteristics.

\subsection{Description of the model}

The finite element model of a high pressure compressor blisk with 36 sectors considered in applications is depicted in Fig. 3. Its modal situation is represented in Fig. 4 where (normalized) eigenfrequencies obtained

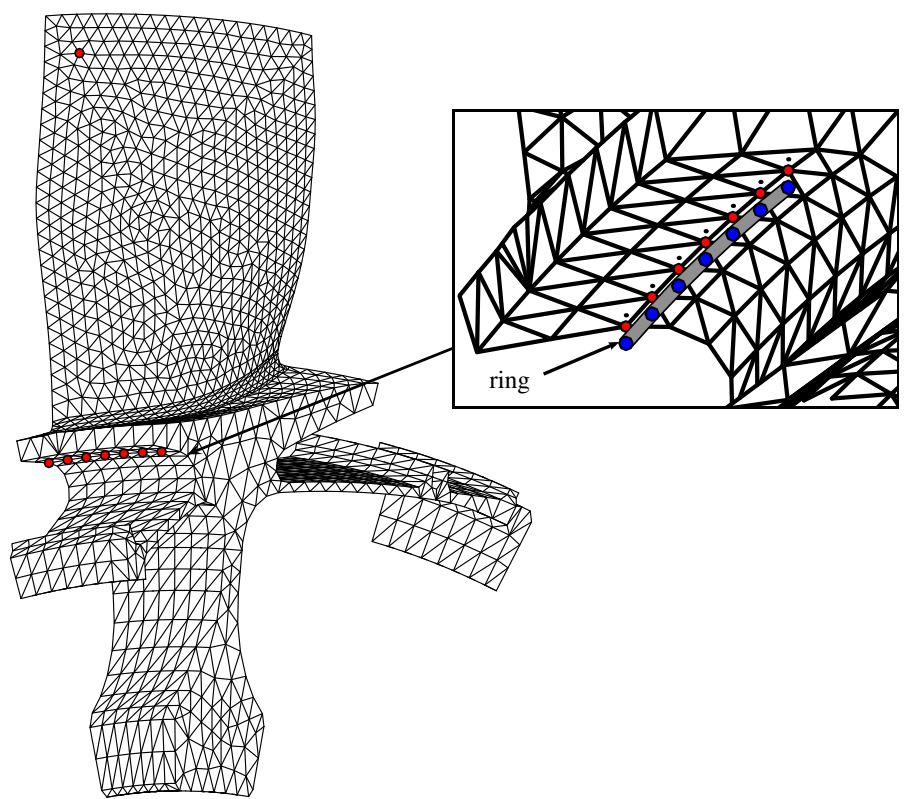

Figure 3: FE model of a blisk (sector) with details of the contact interface and retained nodes

through cyclic symmetry analysis are plotted versus the nodal diameter number (cyclic harmonic). A reducedorder model of the FE model is built using a Craig-Bampton. The set of retained degrees-of-freedom consists in all degrees-of-freedom of nodes at contact interfaces plus those of one node at the top leading edge of the blade (as shown in red in Fig. 3). An harmonic rotating force (with various wave number) is applied to this node and most frequency response results concerns the displacement of this node.

Ring dampers are modeled using three-dimensional beam finite-elements (with six degrees-of-freedom per node) with uniform circular cross section. Design parameters of the ring are its material properties and its outer and inner radii with respect to the axis of rotation. The first radius is defined by the position of the ring on the blisk and the difference between the two radii defines the ring thickness (diameter of the cross section). 


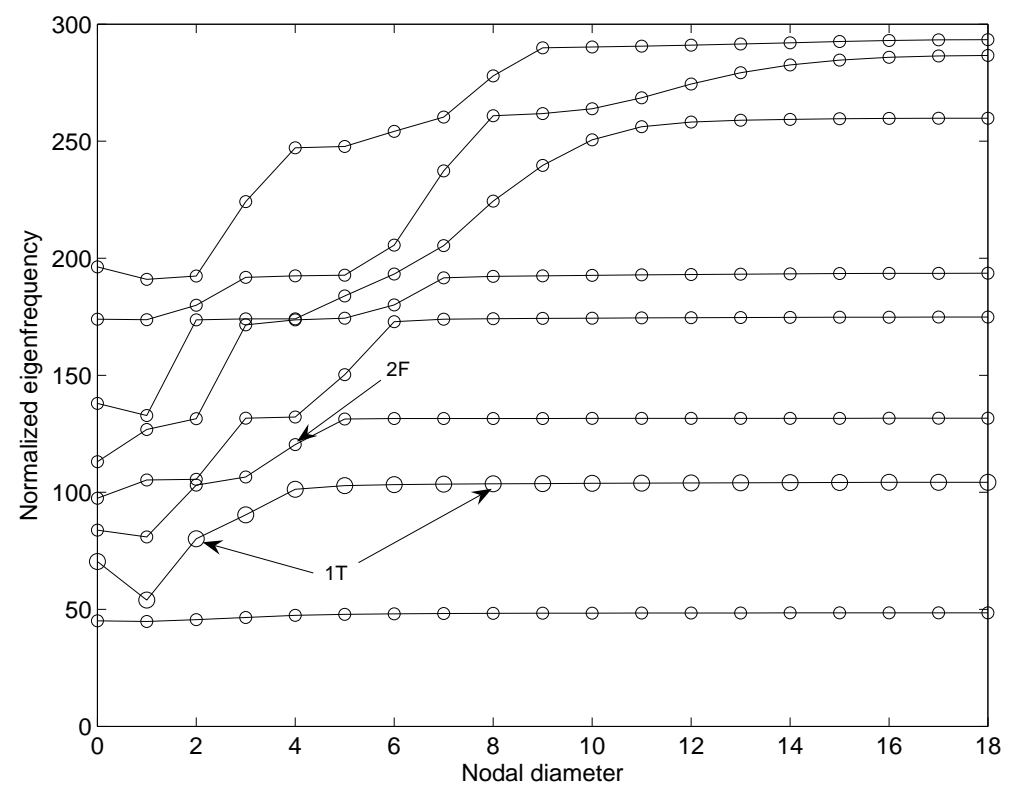

Figure 4: Frequency/Nodal diameters map of the blisk model

Also, the physical implantation of rings requires the latter to be split. As shown in [14], this split essentially induces a symmetry breaking which in turns leads to small perturbations of the structure's dynamics that can be neglected in a first approach. Then, as assumed in the theoretical developments, the ring is assumed to be perfectly symmetric.

A node-to-node contact model is used, assuming that relative motions at contact interfaces are much smaller than distances between adjacent nodes (see Fig. 3). A normal contact preload is applied to each pair of contact nodes to account for centrifugal effects. This preload is typically given by: $m R \Omega^{2}$, in which $m$ is the mass per unit of length of the ring, $R$ is the radius of the neutral axis with respect to the axis of rotation and $\Omega$ is the rotation speed.

Concerning the computation parameters and particularly the number of harmonics, we have used the strategy of optimal determination of the number of harmonics required which depends on the level of nonlinearity in the response, as discussed in the theoretical part. Thus the number of harmonics was dynamically adapted starting with one harmonic and increasing up to seven harmonics near resonances.

\subsection{Effects of blade / disk coupling}

To begin with these numerical applications, we investigate the efficiency of friction ring damping with respect to blade / disk coupling. As rings are physically located underside the disk, their ability to reduce blades vibration is governed by the possible energy transfer from the blade to the disk which is commonly measured by the coupling between the blades and the disk. Preliminary studies [14] (on lumped parameter models) have been shown that this factor was particularly important in this respect and it is then of interest to confirm this observation on more realistic models such as the one consider in this study.

As an example, we focus on the first torsion family modes (with frequencies around 100 UF (Unit of Frequency) in Fig. 4) and consider two excitation configurations which respectively excite harmonics 2 and 8. Figs. 5 show the deformed shapes of respective modes with 2 and 8 nodal diameters which are also pointed in Fig. 4. While these modes feature similar motion of the blade, the two nodal diameter mode has a significantly higher disk participation than the eight nodal diameter one. A ring is introduced at the upstream side of the blisk. Figs. 6 display frequency responses of the nonlinear system at resonances of respective modes for various normal loads applied to the ring. In Fig. 6a, which correspond to a high blade-disk coupling (2 nodal diameters), the resonance amplitude and frequency change significantly as the normal load changes. The higher the normal load is the higher the resonance frequency is. Indeed, high normal loads tend to limit slips at the contact interface whereas lower normal loads make them easier. Sliding contact conditions have a softening effect, hence the decrease of resonance frequencies. In intermediate values of normal loads, the reduction of 


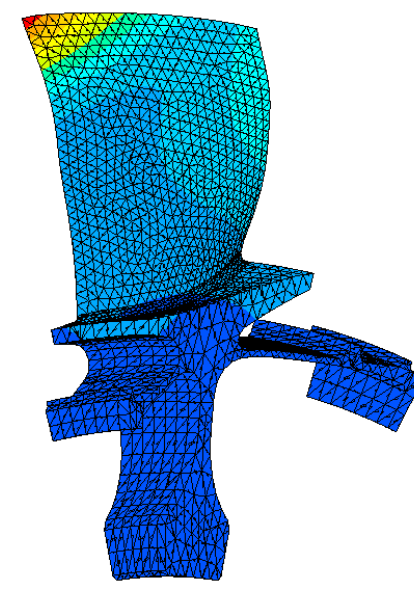

(a)

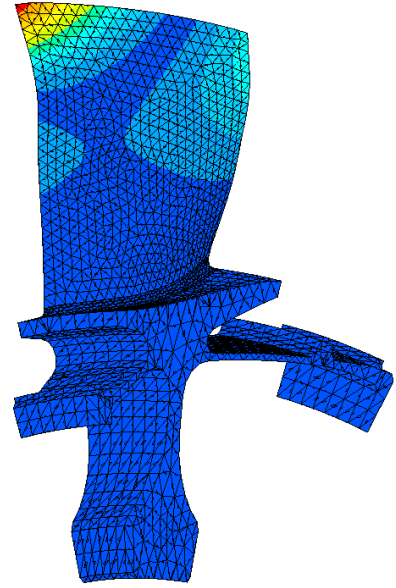

(b)

Figure 5: First torsion mode deformed shapes: (a) two nodal diameters; (b) eight nodal diameters.

the vibratory amplitude is optimal. This maximum efficiency of friction dampers typically corresponds to intermediate stick/slip state; this correlates with many observations on systems involving friction damping (e.g. under-platform dampers for bladed disks assemblies). In contrast, Fig. 6b clearly shows that the ring is less efficient when the blade / disk coupling is less important.

\subsection{Influence of the ring's thickness}

In the second example, we study the influence of ring's thickness which is a major design parameter. In this example we focus on the 3-rd mode at 4 nodal diameters of the blisk (with reference to Fig. 4). This mode, which deformed shape is represented in Fig. 7, can be classified as a second blade bending mode and features an important participation of the disk to the global deformation (i.e. strong coupling).

In Fig. 8, the resonant responses for several ring's thicknesses (D: cross section diameter) are depicted along with the reference resonant response of the blisk without a ring. All other ring's parameters are kept constant. The considered values of the ring thickness are limited in order to keep the ring's mass (proportional to the square of the cross section diameter) in a realistic range in comparison with the blisk mass: the maximum ring's mass does not exceed $0.5 \%$ of the blisk mass. As the damper's thickness increases, the friction damping also increases and an asymptote seams to be reach for high (but still reasonable) values of the ring's thickness. For an extreme high value of the ring thickness (not represented in Fig. 8), the resulting normal contact load (which is proportional to the ring mass) will prevent slips of the ring and thus limit friction damping. This example shows that, for this excitation configuration, the optimal ring thickness appears quite reasonable from a design point of view and an optimal configuration can be find combining both an efficient friction damping and a relative small ring. In this example, the optimal ring's thickness would be around $2 \times D$.

\subsection{Local behavior of the contact interface}

We can examine the local behavior of the contact interface by studying the time histories of the contact variables. We then focus on the resonance point of the frequency response corresponding to the ring's thickness $2 \times D$, located at $116.41 \mathrm{UF}$ in Fig. 8. The time histories (one period) of the contact variables (normal and tangential relative displacements and forces) of a given contact point of the blisk / ring interface are plotted in Figs. 9. First looking at the normal components, we can notice that the relative displacements are very small not more than $10^{-20} \mathrm{~mm}$ and thus very close to the exact solution (zero penetration). This result shows the accuracy of the Dynamic Lagrangian method and contrasts with those that can be obtained using penalty methods which would feature residual penetrations. Also note that the resulting normal force oscillates around a mean value due to the inertia effects. Concerning the tangential components, sticking and sliding phases respectively correspond to nearly constant values of the relative displacement and to nearly constant values of the friction forces are distinguished in Figs. 9b and 9d. The influence of the variable normal contact force 


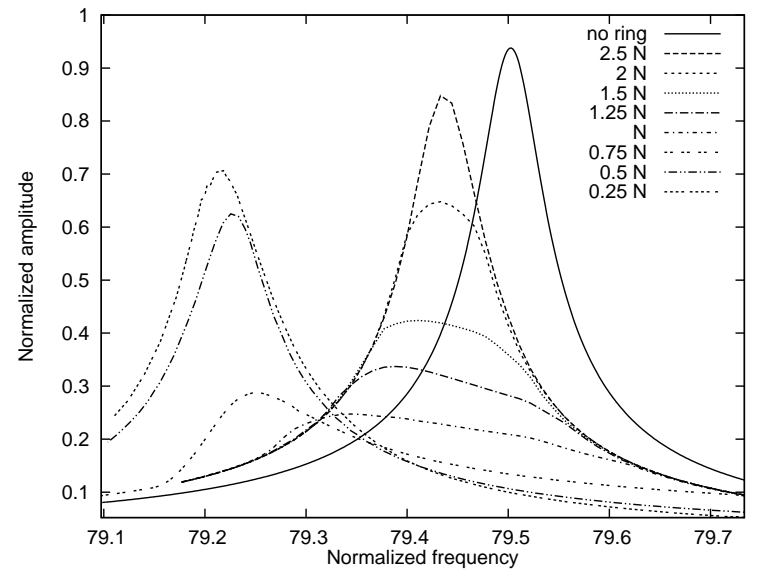

(a) Strong coupling: 1T, two nodal diameters

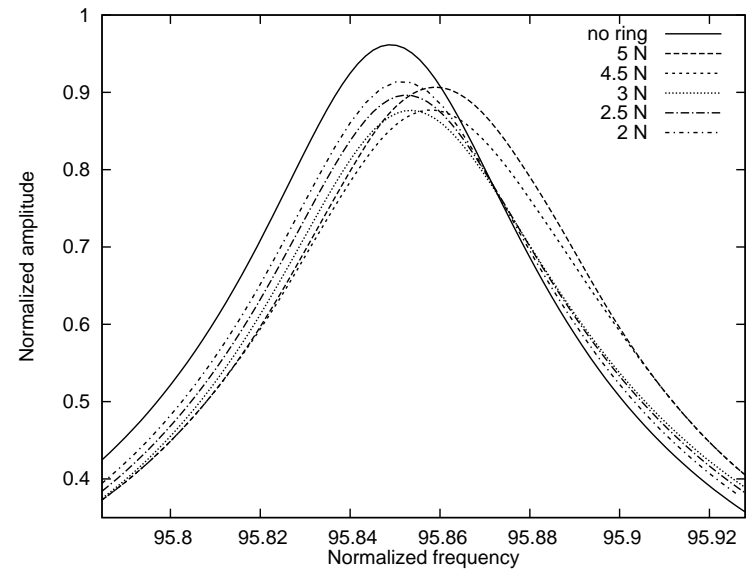

(b) Weak coupling: 1T, eight nodal diameters

Figure 6: Frequency responses for various normal loads $(N)$. Influence of the blade/disk coupling: (a) strong coupling, (b) weak coupling

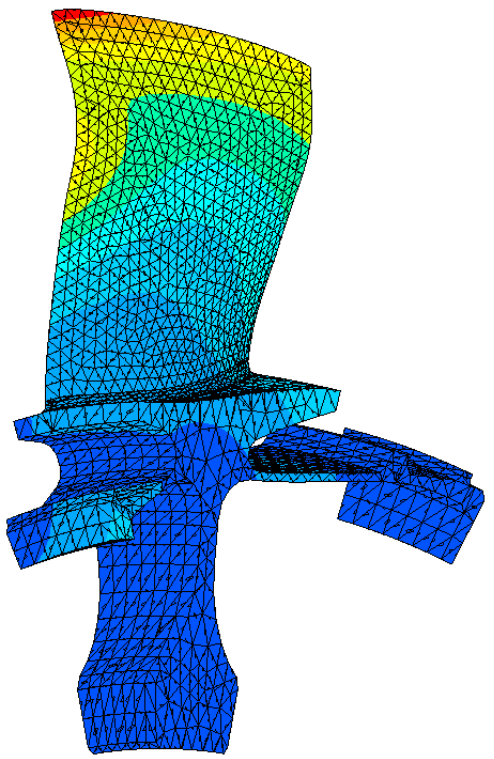

Figure 7: 3-rd mode at 4 nodal diameters; second bending mode. 


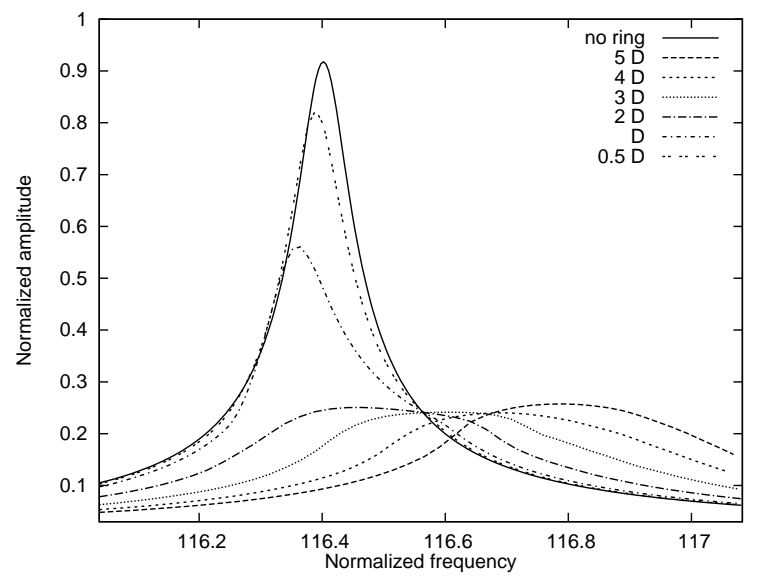

Figure 8: Frequency responses for several (normalized) ring's thicknesses (D); 3-rd mode (2F) at 4 nodal diameters.

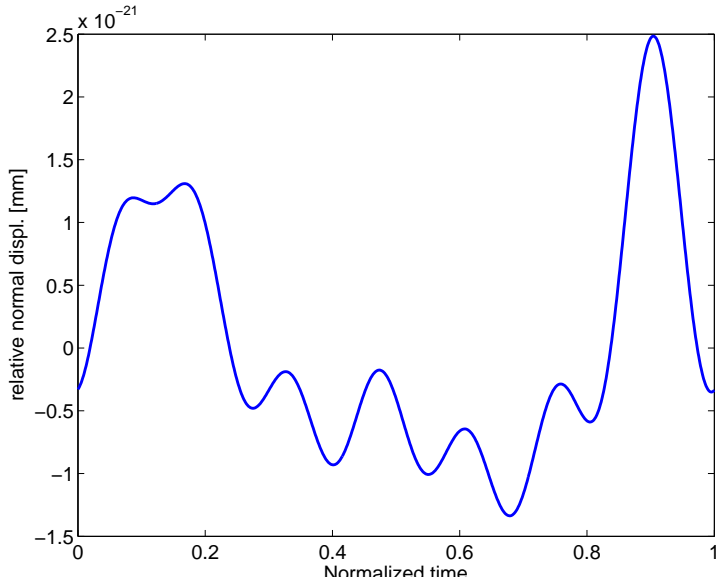

(a)

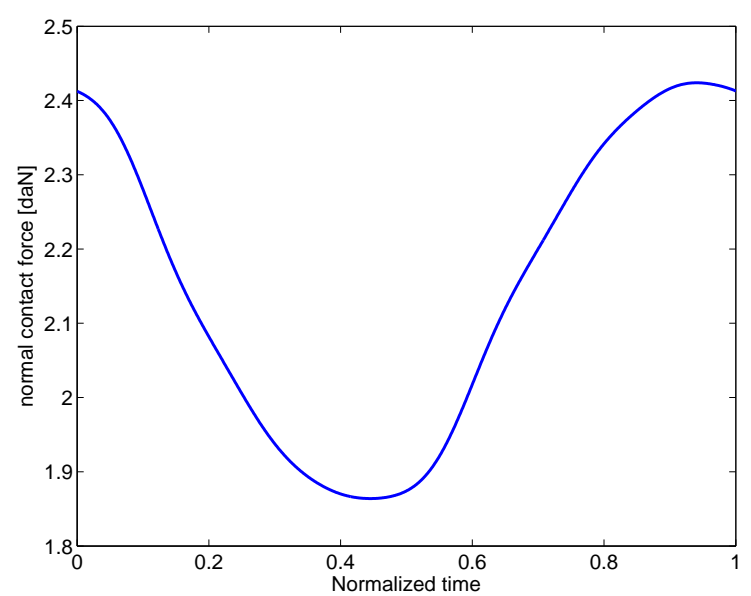

(c)

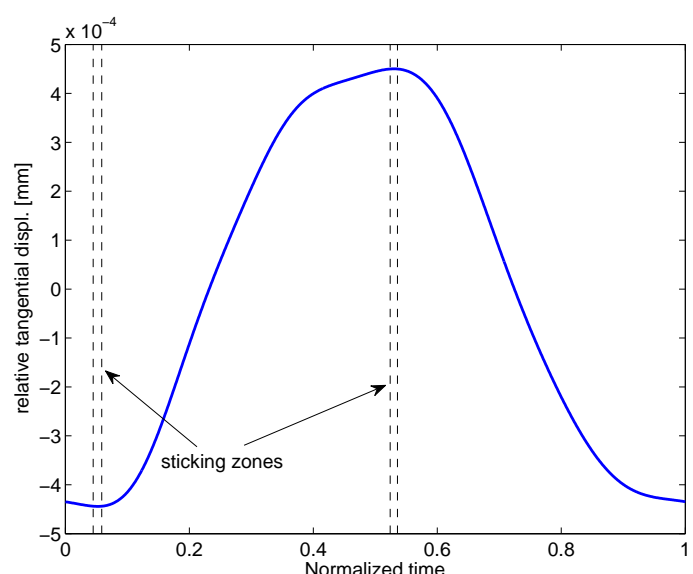

(b)

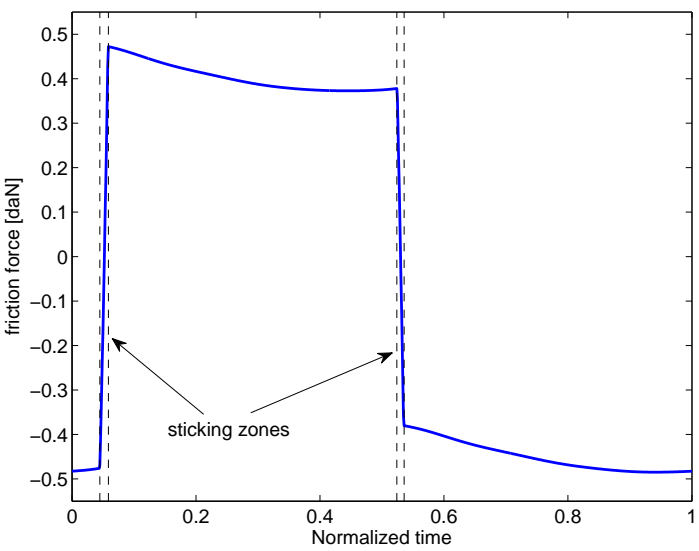

(d)

Figure 9: Local behavior of the contact interface; (a) and (b), normal and tangential relative displacements; (c) and (d), normal and tangential (friction) contact forces 
on the tangential force is noticeable: in the sliding states, the friction force is equal to the Coulomb limiting force which is not constant. Note finally that, as the system's response is in the form of a traveling wave, the spatial distributions of contact forces will look similar to time histories, with a period being defined by the fundamental cyclic harmonic of the response.

\subsection{Using several rings}

In this final example, we consider the implantation of several rings on the blisk. Three possible positions for the rings were chosen, the two firsts are located underside the disk rim (upstream and downstream) and the third is located under the upstream seal.

There are several reasons for using more than one ring on a blisk. First, depending on their location on the disk some rings may be more efficient than other depending on the disk participation at their respective location for a given mode. As an example, let us focus on the 4-th mode at one nodal diameter which is a $1 \mathrm{~T}$ mode with a deformed shape similar to Fig. 5a. In Fig. 10a, the resonant responses of the blisk with each ring

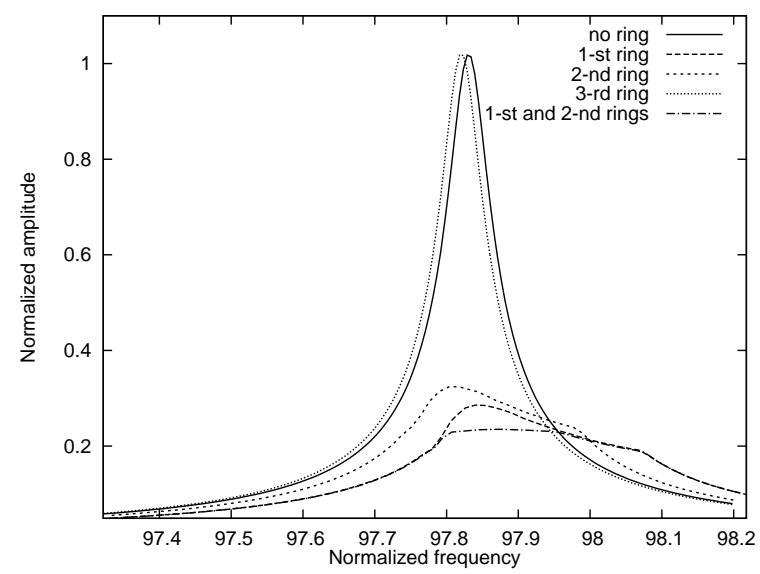

(a) $4^{\text {th }}$ mode $(1 \mathrm{~T})$ at 1 nodal diameter

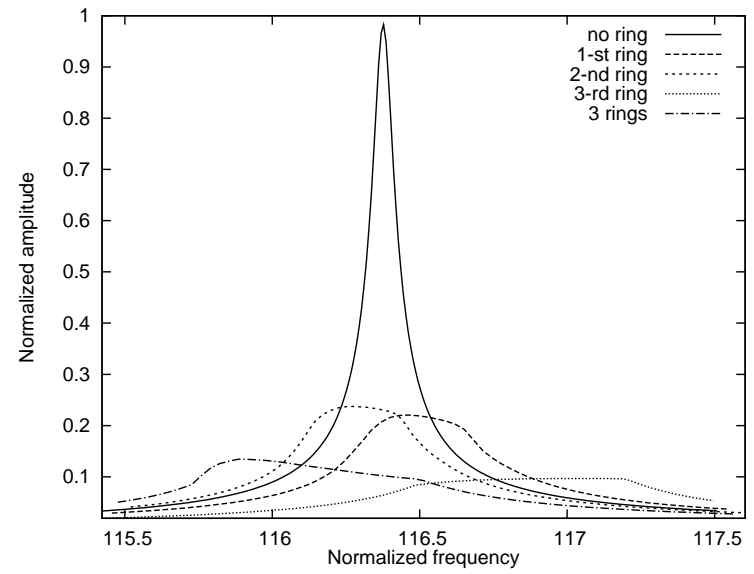

(b) $3^{\text {rd }}$ mode $(2 \mathrm{~F})$ at 4 nodal diameters

Figure 10: Frequency responses for three types of rings

separately are displayed. Clearly, if the two first rings (located under the rim) are efficient for this mode, the third (located underside the seal) is not. Conversely, if we now focus again on the 3-rd mode at four nodal diameters (Fig. 7), the resonant response plotted in Fig. 10b shows that all three rings are efficient and the third one appear more efficient than the others.

The second noticeable reason for using several rings would be to limit the variability of damping efficiency to uncertain parameters (contact or excitation for example). As an example, we study the influence of the level of the excitation still for the 3-rd mode at 4 nodal diameters with fixed values of the contact parameters (normal load and coefficient of friction). The aim is to find the optimal mass (i.e. thickness) of each ring such that each ring works for a different range of excitation force level. Figs. 11 gather the resonant responses for each three ring alone and for all three rings together and for various values of the (normalized) level of excitation $(F)$. The optimal value of the rings' mass repartition is found to be:

- ring 1: $0.56 \times m_{0}$

- $\operatorname{ring} 2: 2.32 \times m_{0}$

- $\operatorname{ring} 3: 0.12 \times m_{0}$

where $m_{0}$ is a normalized mass. The first ring (Fig. 11a) is designed to be efficient around $F$; the second ring (Fig. 11b) is designed to work in the $[2 \times F ; 5 \times F]$ range and the third ring (Fig. 11c) is designed to work in the $[0.5 \times F ; F]$. Consequently when all three rings are introduced the range of efficiency of friction damping encompasses the operating ranges of each ring and spreads from $0.5 \times F$ to $5 \times F$.

Figs. 11 also illustrate how the rings' position affects differently the resonance frequency of the blisk-rings system with reference with the blisk alone. In particular, comparing the resonance frequency of the blisk 


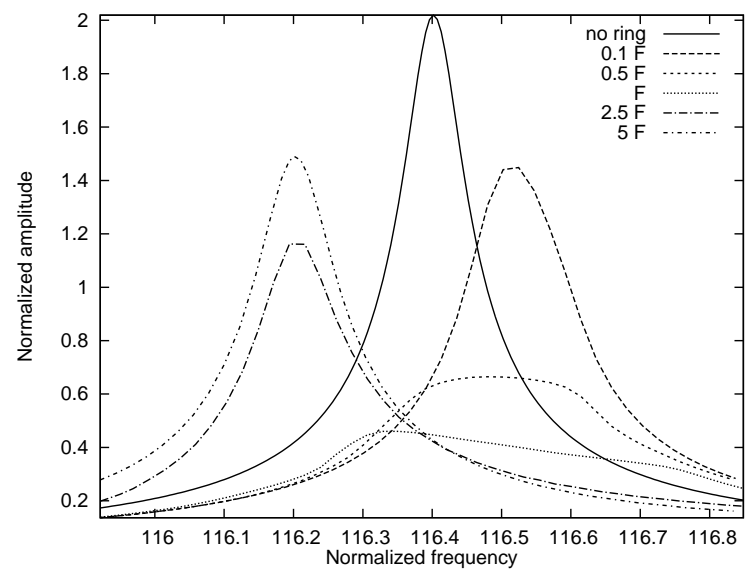

(a) $1^{\text {st }}$ ring only

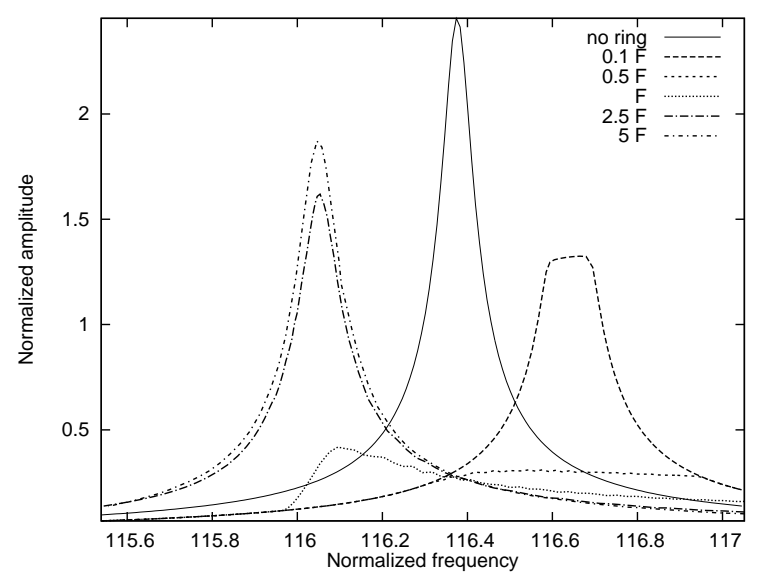

(c) $3^{\text {rd }}$ ring only

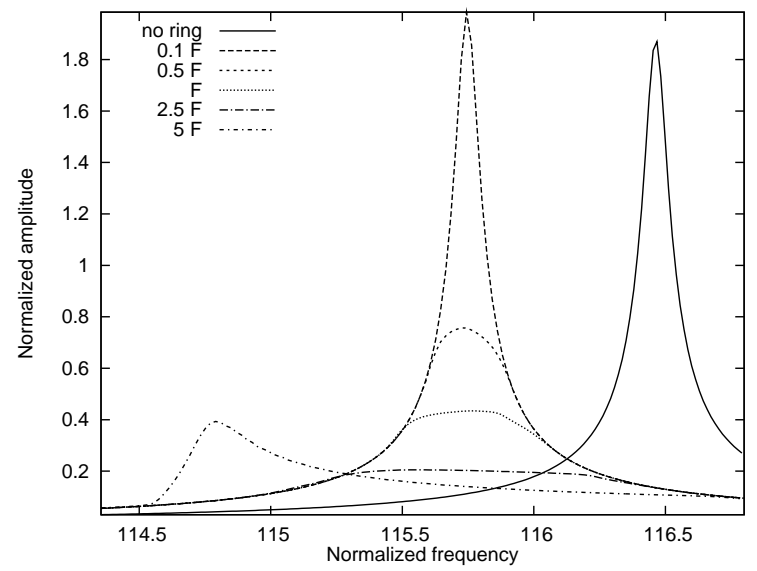

(b) $2^{\text {nd }}$ ring only

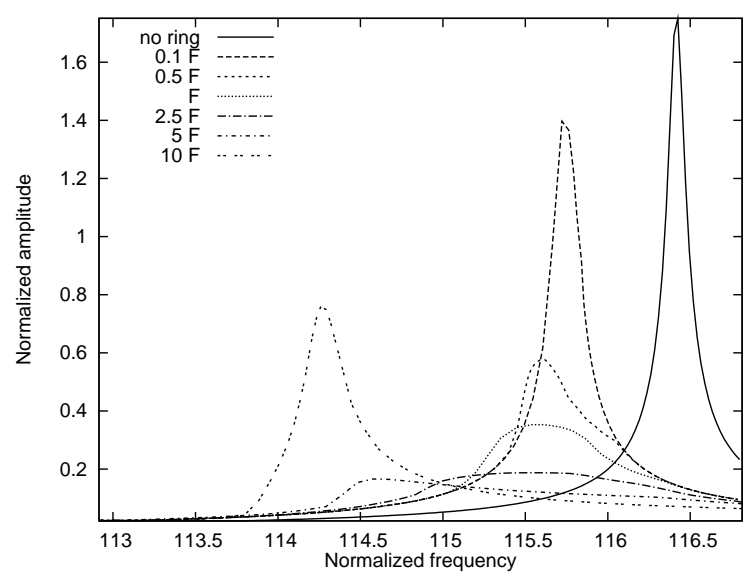

(d) 3 rings together

Figure 11: Frequency responses with single ring or multiple rings, influence of excitation force level $(F)$; 3-rd mode $(2 \mathrm{~F})$ at 4 nodal diameters. 
without a ring with that of the blisk with a ring at the lowest amplitude of excitation $(0.1 \mathrm{~F})$, one can notice that the first and third rings bring stiffness to the system (the resonance frequency with a ring is higher) while the second one has a mass effect conversely.

\section{Conclusions}

A damping strategy for blisks (integrally bladed disks) is investigated; it consists in using small rings located underside the wheel of blisk held in contact due to centrifugal loads. Energy dissipation occurs because of friction phenomena.

A method of analysis of the nonlinear dynamics of the blisk / rings system is presented. In order to determine the steady-state forced response of the nonlinear system to a periodic rotating excitation, an harmonic balance formulation adapted to cyclic structures and rotating excitations is used; this features the use of Dynamic Lagrangian which enforce the contact and friction laws. Condensation procedures on nonlinear and relative degrees-of-freedom is introduced. Also, a strategy for optimal choice of the number of harmonics to be retained is proposed based on the monitoring of an error criterion.

Numerical applications on an industrial scale blisk FE model with friction rings modeled using 3D beam finite-elements are presented. Parameter studies show the efficiency of this damping strategy, depending on the bladed / disk coupling and provide an understanding of the phenomena involved. Optimal performances of friction dampers are typically obtained when the motion of the contact interfaces features alternating stick and slip phases. Design guidelines are drawn from these studies, in particular regarding the range of applications of such damping devices.

\section{Acknowledgment}

Thanks go to Snecma for its technical and financial support. This work takes place in the framework of the MAIA mechanical research and technology program sponsored by CNRS, ONERA and SAFRAN Group.

\section{References}

[1] Griffin, J. H., 1990, "A review of friction damping of turbine blade vibration," International Journal of Turbo and Jet Engines, 7, pp. 297-307.

[2] Sinha, A. and Griffin, J. H., 1983, "Effects of friction dampers on aerodynamically unstable rotor stages," AIAA Journal, 23(2), pp. 262-270.

[3] Csaba, G., 1998, "Forced response analysis in time and frequency domains of a tuned bladed disk with friction dampers," Journal of Sound and Vibration, 214(3), pp. 395-412.

[4] Guillen, J. and Pierre, C., 1999, "An efficient, hybrid, frequency-time domain method for the dynamics of large-scale dry-friction damped structural systems," IUTAM Symposium on Unilateral Multibody Contacts, Dordrecht, Netherlands.

[5] Nacivet, S., Pierre, C., Thouverez, F., and Jézéquel, L., 2003, "A dynamic lagrangian frequency-time method for the vibration of dry-friction-damped systems," Journal of Sound and Vibration, 265, pp. 201-219.

[6] Petrov, E. P., 2003, "Analytical formulation of friction interface elements for analysis of nonlinear multiharmonic vibrations of bladed disks," Journal of Turbomachinery, 125(2), p. 364.

[7] Sanliturk, K. Y. and Ewins, D. J., 1996, "Modelling two-dimensional friction contact and its application using harmonic balance method," Journal of Sound and Vibration, 193(2), pp. 511-523.

[8] Chen, J. J., Yang, B. D., and Menq, C. H., 2000, "Periodic forced response of structures having threedimensional frictional constraints," Journal of Sound and Vibration, 229(4), pp. 775-792.

[9] Menq, C. H., Bielak, J., and Griffin, J. H., 1986, "The influence of microslip on vibratory response, part II: a comparison with experimental results," Journal of Sound and Vibration, 107(2), pp. 295-307. 
[10] Yang, B. D. and Menq, C. H., 1998, "Characterization of contact kinematics and application to the design of wedge dampers in turbomachinery blading: Part 2 - prediction of forced response and experimental verification," Journal of Engineering for Gas Turbines and Power, 120, pp. 418-423.

[11] Berthillier, M., Dupont, C., Mondal, R., and Barrau, J. J., 1998, "Blades forced response analysis with friction dampers," Journal of Vibration and Acoustics, 120, pp. 468-474.

[12] Sanliturk, K. Y., Ewins, D. J., Elliott, R., and Green, J. S., 2001, "Friction damper optimisation: simulation of rainbow tests," Journal of Engineering for Gas Turbines and Power, 123, pp. 930-939.

[13] Niemotka, M. A. and Ziegert, J. C., 1993, "Optimal design of split ring dampers for gas turbine engines," Proceedings of ASME Turbo Expo.

[14] Laxalde, D., Sinou, J. J., Thouverez, F., and Lombard, J. P., 2007, "Qualitative analysis of forced response of blisks with friction ring dampers," European Journal of Mechanics A/Solids, 26(4), pp. 676-687.

[15] Poudou, O. and Pierre, C., 2003, "Hybrid frequency-time domain methods for the analysis of complex structural systems with dry friction damping," Proceedings of 44 th $A I A A / A S M E / A S C E / A H S$ Conferences, Norfolk, Virginia, USA.

[16] Petrov, E. P., 2004, "A method for use of cyclic symmetry properties in analysis of nonlinear multiharmonic vibrations of bladed disks," Journal of Turbomachinery, 126, pp. 175-183.

[17] Thomas, D. L., 1979, "Dynamics of rotationally periodic structures," International Journal for Numerical Methods in Engineering, 14(1), pp. 81-102.

[18] Cameron, T. M. and Griffin, J. H., 1989, "An alternating frequency/time domain method for calculating the steady-state response of nonlinear dynamic systems," Journal of Applied Mechanics, 56, pp. $149-154$.

[19] Petrov, E. P. and Ewins, D. J., 2002, "Models of friction damping with variable normal load for timedomain analysis of vibrations," Proceedings of ISMA - Volume 1, Leuven, Belgium.

[20] Charleux, D., Gibert, C., Thouverez, F., and Dupeux, J., 2006, "Numerical and experimental study of friction damping in blade attachments of rotating bladed disks," International Journal of Rotating Machinery, article ID 71302, 13 pages. 